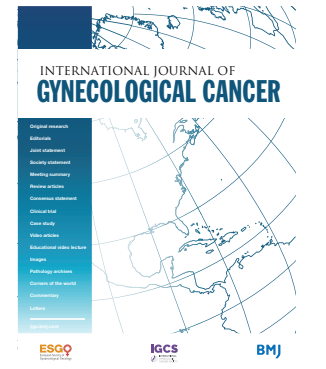
Dr Alejandro Gallego, Department of Medical Oncology, Hospital Universitario La Paz, Madrid CP 28046 , Spain; alex_gallego@hotmail.es

Accepted 27 January 2021
Check for updates

(C) IGCS and ESGO 2021. No commercial re-use. See rights and permissions. Published by BMJ.

To cite: Gallego A, Garrido D, Yébenes L, et al. Int J Gynecol Cancer 2021;31:1292-1296.

\title{
Long-term response to olaparib in BRCA1- related ovarian cancer with brain metastases
}

\author{
Alejandro Gallego \\ Department of Medical Oncology, Hospital Universitario La Paz, Madrid, Spain \\ Diego Garrido \\ Department of Radiology, Hospital Universitario La Paz, Madrid, Spain \\ Laura Yébenes \\ Department of Pathology, Hospital Universitario La Paz, Madrid, Spain \\ Marta Mendiola \\ Translational Oncology Laboratory, IdiPAZ, Hospital Universitario La Paz, Madrid, Spain \\ Beatriz Castelo \\ Department of Medical Oncology, Hospital Universitario La Paz, Madrid, Spain \\ Andres Redondo \\ Department of Medical Oncology, Hospital Universitario La Paz, Madrid, Spain
}

\section{CASE PRESENTATION}

A 51-year-old woman with an unremarkable personal and family medical history sought medical attention after noticing an abdominal mass, which was located mainly in the right iliac fossa. A gynecological ultrasound and a CT body scan showed a $22.3 \times 11.5 \mathrm{~cm}$ pelvic mass dependent on both ovaries, with peritoneal implants, retroperitoneal lymph nodes, and ascites, without supra-diaphragmatic disease, associated with a serum CA125 level of $2634 \mathrm{kIU} / \mathrm{L}$. The patient first underwent an exploratory laparoscopy, and later a complete cytoreduction was performed with hysterectomy, bilateral salpingo-0ophorectomy, omentectomy, appendectomy, pelvic and para-aortic lymphadenectomy, and resection of tumor implants and the transverse, descending, and sigmoid colon. The histopathological examination confirmed the diagnosis of International Federation of Gynecology and Obstetrics (FIGO) stage IIIC high-grade serous ovarian carcinoma (Figure 1). After primary cytoreduction, the patient received six cycles of carboplatin, area under the curve $6 \mathrm{mg} / \mathrm{mL} / \mathrm{min}$, and paclitaxel $175 \mathrm{mg} / \mathrm{m}^{2}$ every 3 weeks. At the end of the treatment no disease remained on the CT scan and the CA125 level was normal.

Meanwhile, a genetic study of germline BRCA1/2 was performed, in which a pathogenic BRCA1 mutation was discovered. For this reason, after chemotherapy was completed the patient was eligible to participate in the SOLO-1 clinical trial, a double-blind study in which patients were randomized to receive olaparib (400 mg twice daily) or placebo for 2 years. A total of 17 months after beginning study treatment, the patient was admitted to the emergency room due to intracranial hypertension symptomatology with

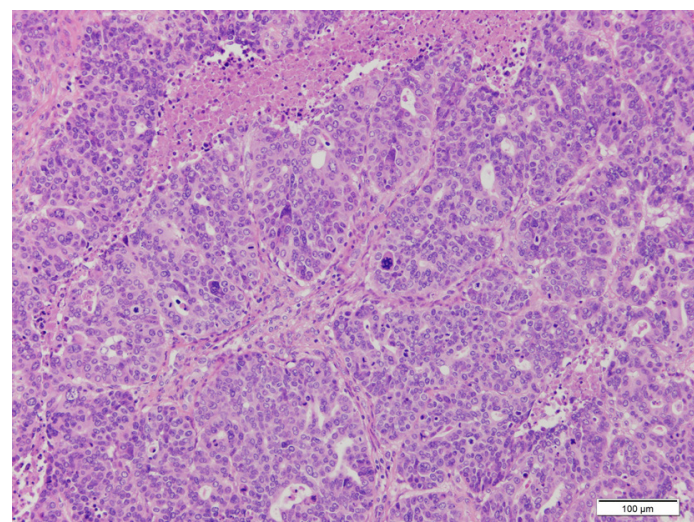

Figure $1 \mathrm{He}, 20 x$. High-grade serous carcinoma of the ovary. Displays slit-like spaces and solid pattern, marked cytologic atypia, necrosis, and high mitotic activity.

vomiting, blurred vision, and headache that did not improve with analgesia. The cerebral scan perfomed in September 2016 showed multiple brain recurrence (Figure 2). The CT body scan showed mediastinal lymph nodes suspected of being malignant. No disease in the abdomen or pelvis was identified.

The patient underwent whole brain radiotherapy, with a total dose of 30 Gy delivered in 10 fractions over the course of 10 days. The patient then started treatment with carboplatin, area under the curve $4 \mathrm{mg} / \mathrm{mL} / \mathrm{min}$, on day 1 and gemcitabine $1000 \mathrm{mg} / \mathrm{m}^{2}$ on days 1 and 8 every 3 weeks. After three cycles of chemotherapy, a further decrease in the size of the cerebral lesions was seen in December 2016. For this reason, the blinding of the SOLO-1 study was removed, so that she could be prescribed a poly (ADPribose) polymerase (PARP) inhibitor in the event that 


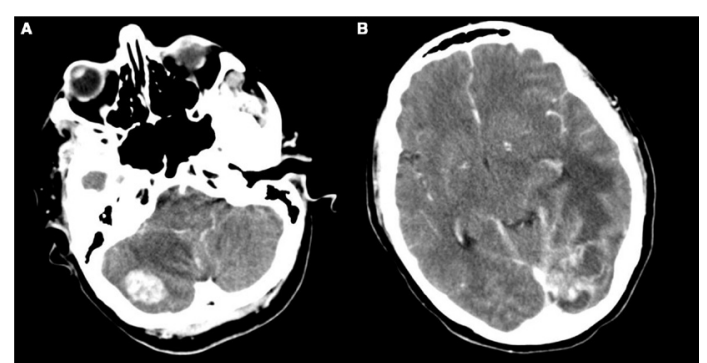

Figure 2 Brain metastases (September 2016). (A) Axial contrast-enhanced CT brain scan through the posterior cranial fossa revealing an enhanced heterogeneous lesion measuring $2.5 \mathrm{~cm}$ surrounded by vasogenic edema in the right cerebellar hemisphere. (B) Axial contrast-enhanced CT brain scan showing a ring-enhancing lesion with a cysticnecrotic component and a solid area with heterogeneous enhancement surrounded by vasogenic edema in the left occipital hemisphere.

she had received the placebo. Due to the hematologic toxicity, with grade 4 thrombocytopenia, the patient was admitted to the hospital and the last chemotherapy cycle was canceled after the administration of five cycles. At this time, March 2017, a cerebral MRI was performed, where a decrease in the size of the lesions was seen. Once it was confirmed that the patient had received the placebo in the clinical trial, olaparib was started in April 2017 at a dose of $400 \mathrm{mg}$ twice a day (capsule dose). Her tolerance to treatment was appropriate except for two episodes of grade 3 neutropenia, which led to two dose reductions. Since August 2017 the patient has been taking $200 \mathrm{mg}$ twice a day. In the first MRI performed 3 months after the initiation of olaparib, July 2017, radiological improvement was noted, with a reduction in the size of the lesions. The main lesion had decreased from 2.9 to $2.2 \mathrm{~cm}$.

During the course of the next 30 months, subsequent MRls continued to show a slow reduction in the brain metastases, achieving a partial tumor response. On July 11, 2019, the formulation was changed and the patient began receiving a $150 \mathrm{mg}$ tablet twice a day. No noteworthy changes in tolerance or in response were seen. At the present time, in October 2020, more than 4 years after finishing radiotherapy treatment and 42 months after beginning olaparib, the response achieved in the brain metastases has been maintained without additional extracranial tumor lesions (Figure 3). Moreover, her CA125 level is within the normal range and, perhaps even more important, she continues to tolerate the treatment well and has good general health: her dizziness has improved and her Eastern Cooperative Oncology Group (ECOG) performance status is 0 .

In September 2020, the patient was also diagnosed with luminal A breast cancer, T1N0 (Figure 4), and she will undergo surgery with a wide excision lumpectomy and sentinel lymph node biopsy.

\section{Dr Garrido: How often are brain metastases diagnosed in ovarian cancer?}

Ovarian cancer is a rare cause of brain metastases. The reported average incidence is estimated to be approximately $1-2.5 \%$, with the cerebellum the most common site of metastases. ${ }^{12}$ The majority of patients have multiple brain lesions, and some $49.8 \%$ also present extracranial metastases at the time of diagnosis. The most frequent histologic sub-type associated with brain metastases

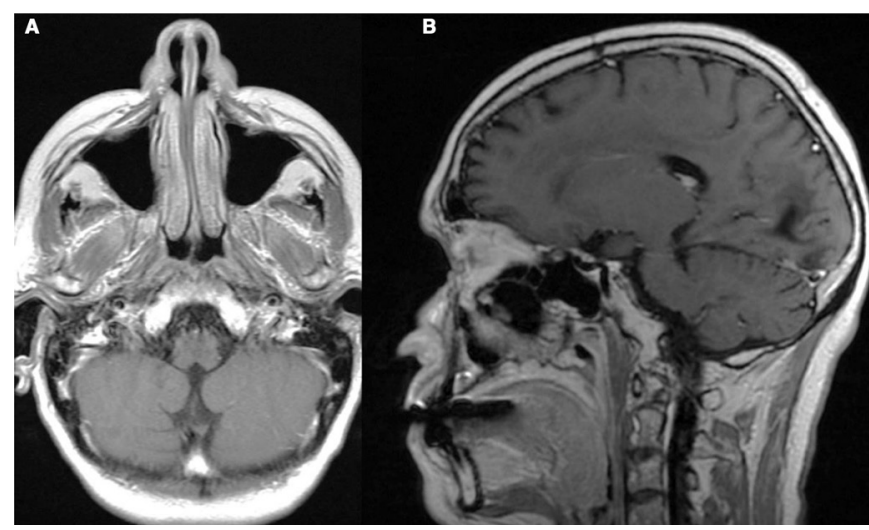

Figure 3 Complete response of one of the brain metastases (August 2020). (A) Axial post-contrast T1weighted brain MRI through the posterior cranial fossa showing a focal hypointense non-enhancing area at the right hemisphere consistent with post-treatment gliosis. (B) Axial post-contrast T1-weighted brain MRI at the level of the left occipital lobe revealing a dilated occipital horn of the lateral ventricle with an extensive focal hypointense non-enhancing area compatible with encephalomalacia and gliosis.

is high-grade serous carcinoma, with an average survival in those patients with multiple brain metastases of 9.2 months (compared with 21.4 months for single lesions). ${ }^{1}$ Although these are the most comprehensive available data regarding the survival of patients with ovarian cancer and brain metastases, it is important to clarify that the survival of these patients was analyzed in the pre-PARP inhibitors' era.

\section{Dr Mendiola: Could BRCA status influence the development of brain metastases in ovarian cancer? and homologous recombination deficiency?}

The rate of germline and somatic BRCA1/2 mutations among patients with high-grade serous carcinoma is between $20 \%$ and $30 \%$. Moreover, in recent years, more genes related to hereditary breast and ovarian cancer syndrome have been discovered. Some of these genes, such as PALB2, BRIP1, RAD51C and RAD51D, increase the risk of ovarian cancer. However, we do not yet understand the

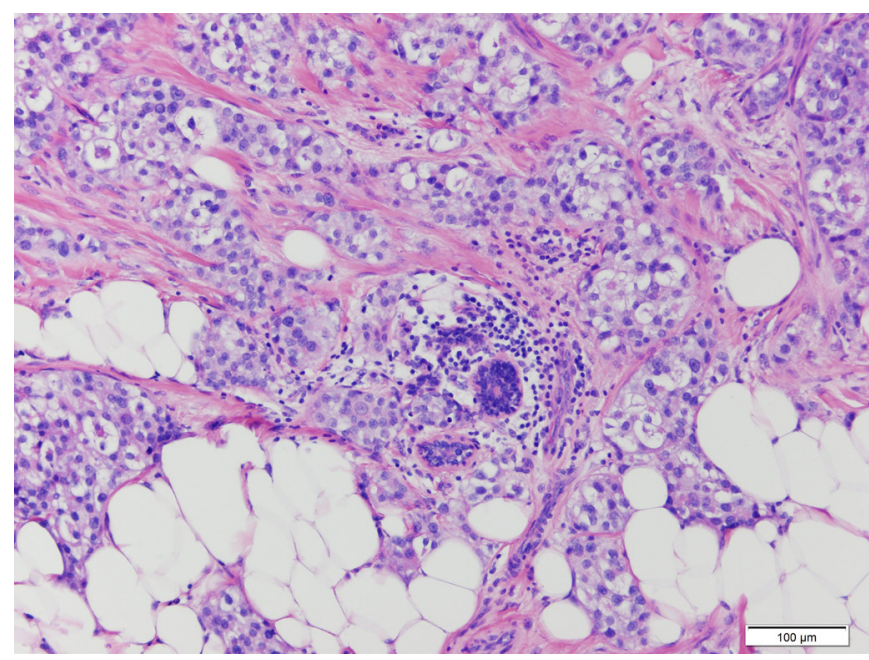

Figure $4 \mathrm{He}, 20 \mathrm{x}$. Invasive breast carcinoma of no special type, with glandular differentiation. Nottingham grade 2 . 


\section{Case study}

influence of these newly discovered genes on the evolution of ovarian cancer.

Patients with ovarian cancer with a BRCA1/2 mutation have a higher rate of brain metastases than those patients without the mutation: $3 \%$ and $0.6 \%$, respectively, ${ }^{2}$ as occurs in BRCA1/2related breast cancer, regardless of the stage, estrogen receptor, or HER2 expression. ${ }^{3}$

In addition to BRCA1/2, other mechanisms involved in DNA repair, such as a deficiency in the homologous recombination system (HRD), could play an important role in the development of brain metastases. Diossy $M$ et al and Sun J et al described an increase in genomic instability based on HRD in brain metastases in relation to primary breast cancer and colorectal cancer. ${ }^{45}$ Some mechanisms have been proposed, such as the ability of tumor cells with higher $H R D$ to adjust more readily to a distinct environment like the brain.

Approximately half of patients with ovarian cancer have HRD, and this percentage might be higher in those who have brain metastases, as occurs in breast and colorectal cancer. Since, not all HRD alterations increase the risk of developing breast cancer, this might explain the clinical case presented, with the patient continuing to have a response to the brain metastases while also developing breast cancer.

Dr Castelo: What is the role of PARP inhibitors as maintenance treatment in recurrent BRCA1/2-related ovarian cancer? How many of these patients are long-term responders?

Currently, several PARP inhibitors, such as olaparib, niraparib, and rucaparib, have shown a significant clinical benefit in patients with high-grade serous ovarian cancer, particularly in BRCA1/2-related ovarian cancer, when used as both a single agent and as maintenance treatment after platinum in recurrent ovarian cancer. The results of the SOLO-2 clinical trial showed a significant improvement in the median progression-free survival for the olaparib arm over placebo of 13.6 months (median progression-free survival: 19.1 vs 5.5 months, respectively) in patients with platinum-sensitive recurrent BRCA1/2 mutated ovarian cancer with a complete or partial response to platinum-based chemotherapy. ${ }^{6}$ To date, the progression-free survival of the patient reported here (40 months) is twice as long as the median progression-free survival reported in SOLO-2. Final overall survival results for this study have recently been reported, with a median overall survival of 38.8 months in placebo group vs 51.7 months in olaparib group. ${ }^{7}$ In study 19 clinical trial, there were also long-term responders to olaparib: $10.8 \%$ of patients with BRCA1/2 mutation had been undergoing treatment for longer than 6 years. ${ }^{8}$ However, due to the rarity of brain metastases in patients with ovarian cancer and the fact that the SOLO-2 clinical trial did not admit patients with symptomatic uncontrolled brain metastases, as far as we know, our patient with ovarian cancer and brain metastases has had the longest response reported to date to a PARP inhibitor.

\section{Dr Redondo: Are PARP inhibitors an effective treatment for the central nervous system? What other treatments could be effective?}

The blood-brain barrier, a physiological barrier whose goal is to protect the central nervous system from exogenous factors, remains a classic sanctuary of the human body. In combination with poor perfusion of blood vessels, it prevents most treatments from reaching an effective concentration in the central nervous system. This barrier is one of the reasons why the treatment of choice for some patients with brain metastases is radiotherapy, or whole-brain radiotherapy. Chemotherapy treatments that are able to enter the blood-brain barrier include alkylating liposoluble agents, such as temozolomide, nitrosoureas, and platinum salts. ${ }^{9}$ Currently, for patients who are expected to have prolonged survival, the most common approach is to seek treatment alternatives to whole-brain radiotherapy, with the aim of preventing longterm adverse effects. ${ }^{10}$ The new radiotherapy techniques, such as intensity-modulated radiation therapy and stereotactic radiotherapy, lead to greater tumor control with fewer consequences. In patients with single brain metastases or in those cases where tissue is required, surgery should also be considered. On the other hand, in some asymptomatic patients with multiple brain metastases, systemic treatment could be considered in order to avoid radiotherapy sequelae. This therapeutic approach is already being performed with other types of tumors with a higher incidence of brain metastases (and greater treatment experience), including patients with non-small cell lung cancer with a driver mutation, such as an epidermal growth factor receptor mutation or anaplastic Iymphoma kinase rearrangement. ${ }^{11}$

Radiation can also disrupt the blood-brain barrier, could enable more successful delivery of other chemotherapy agents, and might also enhance the efficacy of PARP inhibitors, such as veliparib. ${ }^{12}$ However, a randomized phase II trial of veliparib, radiotherapy, and temozolomide in patients with unmethylated methylguanine-DNA methyltransferase glioblastoma did not improve progression-free survival. ${ }^{13}$ Moreover, not all PARP inhibitors have the same characteristics and thus could have varying success in crossing the blood-brain barrier. In the case of another PARP inhibitor, niraparib, one study reported an increase in the brain concentration and intracranial tumor response in a rat model with a BRCA2-mutant xenograft treated with niraparib. The conclusion of that study was that niraparib has sufficient exposure in rodent brains to have therapeutic benefits in an intracranial BRCA-mutant human xenograft model. ${ }^{14}$

There is scant evidence of the effectiveness of olaparib in patients with ovarian cancer and brain metastases. In the Olympiad clinical trial, a phase III, randomized study that enrolled patients with germline BRCA1/2 mutated and HER2-negative metastatic breast cancer, they found that olaparib could have the same effectiveness (objective response rate) in brain metastases as in lung and liver metastases. ${ }^{15}$ However, these results should be interpreted with caution due to the limited number of patients and the lack of information on previous treatments, such as surgery or brain radiotherapy. Furthermore, one case report reported on the effectiveness of olaparib in a patient with leptomeningeal disease of ovarian cancer, also after radiotherapy treatment, in this case 20 Gy distributed in five fractions; the progression-free survival was only 12 months. ${ }^{16}$

Dr Redondo: What percentage of patients with BRCA1/2 mutated ovarian cancer will develop secondary tumors? What would be the follow-up recommendations for patients with recurrent ovarian cancer?

The cumulative lifetime risk of breast and ovarian cancer to 80 years is about $72 \%$ and $44 \%$ in BRCA1 and $69 \%$ and $17 \%$ in 
BRCA2 carriers, respectively. The incidence increases rapidly until age 30 to 40 for BRCA1 and until age 40 to 50 for BRCA2 carriers. However, the risk of developing ovarian cancer under the age of 40 , even in BRCA1/2 carriers, is low, and the prognosis for these patients is poorer. ${ }^{17}$ On the other hand, previous data have suggested that salpingo-oophorectomy might reduce the risk of breast cancer, although findings from a prospective study suggest a role for this surgery in the prevention of pre-menopausal breast cancer in BRCA2, but not in BRCA1 carriers. ${ }^{18}$ For all these reasons, women are less likely to develop breast cancer after ovarian cancer than the other way around, even though the exact incidences are unknown. In the retrospective study carried out by Ratner et al, only 95 of the 4515 (2.1\%) patients with ovarian cancer included were later diagnosed with breast cancer. ${ }^{2}$ The risk of other cancers, such as pancreatic cancer or melanoma, should also be taken into account, particularly in BRCA2 mutation carriers with a family history of these malignancies.

The best screening strategy for breast cancer follow-up in BRCA1/2 patients with recurrent ovarian cancer is also controversial. With a few exceptions, prophylactic bilateral mastectomy would not be recommended for these patients, and breast cancer screening should be individualized in accordance with the Familial Cancer Unit, based on the ovarian cancer prognosis, the patient's age, and her family history. A suggestion for breast cancer screening in these patients, particularly in those with platinumsensitive recurrent ovarian cancer, is to perform a mammography and breast examination every 6-12 months. Given the mortality, the impact of adding breast MRI to the screening of BRCA1/2 mutation carriers is not completely clear, we suggest performing this imaging test only in selected cases.

\section{CLOSING SUMMARY}

Patients with BRCA1/2-related ovarian cancer have been reported to have better prognoses and a better treatment response, particularly to platinum-based chemotherapy and PARP inhibitors. However, these tumors could display more aggressive behavior and there is a greater risk of developing brain metastases than in those without a BRCA1/2 mutation. However, the incidence of brain metastases in patients with ovarian cancer is low, and the best treatment for these cases is still controversial.

A combination of surgery, whole-brain radiotherapy, and chemotherapy for the treatment of brain metastases in patients with ovarian cancer might be associated with longer survival than treatment with whole-brain radiotherapy alone. The reported median survival for these patients in the study of Pakneshan $S$ et al was 20.5 months and 9.1 months, respectively, even in those patients with platinum-sensitive recurrence and BRCA1/2-related ovarian cancer. ${ }^{1}$ Due to the long-term effects of whole-brain radiotherapy, we should prioritize other techniques that modulate irradiation, such as intensity-modulated radiation therapy and stereotactic radiotherapy.

On the other hand, we also have systemic treatments, such as platinum chemotherapy and PARP inhibitors, which can cross the blood-brain barrier and be effective in the central nervous system. Moreover, there is a clinical rationale that suggests that brain metastases might have a better response to PARP inhibitors, which could be related to a higher homologous recombination system. This effectiveness could be even higher in patients with BRCA1/2 mutations. In the clinical case reported here, the good response to systemic treatment, with a reduction in brain lesions, was observed more than 2 years after completing radiotherapy. For all these reasons, the option of administering systemic therapy with platinum-based chemotherapy followed by a PARP inhibitor can be considered for selected asymptomatic patients, instead of other surgical or radiotherapeutic approaches. However, it should be noted that we have limited experience with this therapeutic approach.

Further studies are warranted to unravel the mechanisms involved in the development of brain metastases in patients with recurrent ovarian cancer, and the most appropriate treatments. The role of PARP inhibitors, alone, sequential, or in combination with brain radiotherapy, is also unclear. Due to this low incidence, the chances of developing a clinical trial are also low, which is why it is even more important to report clinical cases and make efforts to carry out international registries, to improve our knowledge in this field. Moreover, patients with ovarian cancer and controlled brain metastasis should not be excluded for clinical trials, in order to learn more about the behavior of the new drugs studied in these patients.

Contributors Presenter: AG; discussants: DG, MM, BC, AR; pathologist: LY.

Funding The authors have not declared a specific grant for this research from any funding agency in the public, commercial or not-for-profit sectors.

Competing interests AG: reports speaker bureau/expert testimony (Clovis, Merck Sharp \& Dohme, AstraZeneca, GSK, PharmaMar, and Roche) and travel/ accommodation/expenses (Merck Sharp \& Dohme, PharmaMar, Roche, Eisai, Pfizer Pierre-Fabre, and Tesaro-A GSK Company). MM: reports speaker bureau/expert testimony (GSK, MSD), research grant/funding to his institution (Eisai), and travel/ accommodation/expenses (Pharmamar, Pfizer, GSK, Eisai). AR: reports honoraria and advisory/consultancy (MSD, AstraZeneca, Roche, GSK, Clovis, PharmaMar, Lilly, Amgen), research grant/funding to his institution (Eisai, PharmaMar, Roche), travel/ accommodation/expenses (AstraZeneca, Tesaro, PharmaMar, Roche), and speakers bureau (MSD, AstraZeneca, Roche, GSK, Clovis, PharmaMar), outside the submitted work.

Patient consent for publication Not required.

Provenance and peer review Commissioned; externally peer reviewed.

\section{REFERENCES}

1 Pakneshan S, Safarpour D, Tavassoli F, et al. Brain metastasis from ovarian cancer: a systematic review. J Neurooncol 2014;119:1-6.

2 Ratner E, Bala M, Louie-Gao M, et al. Increased risk of brain metastases in ovarian cancer patients with BRCA mutations. Gynecol Oncol 2019;153:568-73.

3 Zavitsanos PJ, Wazer DE, Hepel JT, et al. BRCA1 mutations associated with increased risk of brain metastases in breast cancer: a 2:1 matched-pair analysis. Int J Radiat Oncol Biol Phys 2016;96:S59-60.

4 Diossy M, Reiniger L, Sztupinszki Z, et al. Breast cancer brain metastases show increased levels of genomic aberration-based homologous recombination deficiency scores relative to their corresponding primary tumors. Ann Oncol 2018;29:1948-54.

5 Sun J, Wang C, Zhang Y, et al. Genomic signatures reveal DNA damage response deficiency in colorectal cancer brain metastases. Nat Commun 2019;10:3190.

6 Pujade-Lauraine E, Ledermann JA, Selle F, et al. Olaparib tablets as maintenance therapy in patients with platinum-sensitive, relapsed ovarian cancer and a BRCA1/2 mutation (SOLO2/ENGOT-Ov21): a double-blind, randomised, placebo-controlled, phase 3 trial. Lancet Oncol 2017;18:1274-84.

7 Poveda A, Floquet A, Ledermann JA, et al. Final overall survival (OS) results from SOLO2/ENGOT-ov21: a phase III trial assessing maintenance olaparib in patients (PTS) with platinum-sensitive, relapsed ovarian cancer and a BRCA mutation. JCO 2020;38. 


\section{Case study}

8 Gourley C, Friedlander M, Matulonis UA, et al. Clinically significant long-term maintenance treatment with olaparib in patients (PTS) with platinum-sensitive relapsed serous ovarian cancer (PSR SOC). JCO 2017;35:5533.

9 Deeken JF, Löscher W. The blood-brain barrier and cancer: transporters, treatment, and Trojan horses. Clin Cancer Res 2007;13:1663-74.

10 Tallet AV, Azria D, Barlesi F, et al. Neurocognitive function impairment after whole brain radiotherapy for brain metastases: actual assessment. Radiat Oncol 2012;7.

11 Lin JJ, Jiang GY, Joshipura N, et al. Efficacy of alectinib in patients with ALK-positive NSCLC and symptomatic or large CNS metastases. J Thorac Oncol 2019;14:683-90.

12 Barazzuol L, Jena R, Burnet NG, et al. Evaluation of poly (ADPribose) polymerase inhibitor ABT-888 combined with radiotherapy and temozolomide in glioblastoma. Radiat Oncol 2013;8.

13 Khasraw M, McDonald KL, Rosenthal M, et al. A randomized phase II trial of veliparib (V), radiotherapy (RT) and temozolomide (TMZ) in patients (PTS) with unmethylated MGMT (UMGMT) glioblastoma (GBM). JCO 2019;37:2011.
14 Mikule K, Wilcoxen K. Abstract B168: the PARP inhibitor, niraparib, crosses the blood brain barrier in rodents and is efficacious in a BRCA2-mutant intracranial tumor model. AACR-NCIEORTC International Conference: Molecular Targets and Cancer Therapeutics, Boston, MA, 2015.

15 Nadine T, Seock-Ah I, Senkus-Konefka E. Olaparib versus chemotherapy treatment of physician's choice in patients with a germline BRCA mutation and HER2-negative metastatic breast cancer (OlympiAD): efficacy in patients with visceral metastases. JCO 2018;36.

16 Bangham M, Goldstein R, Walton $\mathrm{H}$, et al. Olaparib treatment for BRCA-mutant ovarian cancer with leptomeningeal disease. Gynecol Oncol Rep 2016;18:22-4.

17 Kuchenbaecker KB, Hopper JL, Barnes DR, et al. Risks of breast, ovarian, and contralateral breast cancer for BRCA1 and BRCA2 mutation carriers. JAMA 2017;317:2402-16.

18 Kotsopoulos J, Huzarski T, Gronwald J. Bilateral oophorectomy and breast cancer risk in BRCA1 and BRCA2 mutation carriers. J Nat Cancer Inst 2016;109:djw177. 